Article

\title{
Post-Vaccination Yellow Fever Antibodies Enhance Zika Virus Infection in Embryoid Bodies
}

\author{
Emily M. Schultz, TyAnthony J. Jones and Kelli L. Barr* \\ Department of Biology, Baylor University, Waco, Texas and USA; Emily_Schultz1@baylor.edu; \\ Ty_jones1@baylor.edu \\ * Correspondence: kelli_barr@baylor.edu
}

\begin{abstract}
Zika virus (ZIKV) is a flavivirus that originated in Africa but emerged in Latin America in 2015. In this region, other flaviviruses such as Dengue (DENV), West Nile, and Yellow Fever Virus (YFV) also circulate, allowing for possible antigenic cross-reactivity to impact viral infections and immune responses. Studies have found antibody mediated enhancement between DENV and ZIKV, but the impact of YFV antibodies on ZIKV infection has not been fully explored. ZIKV infections cause congenital syndromes, such as microcephaly, necessitating further research into ZIKV vertical transmission through the placental barrier. Recent advancements in biomedical engineering have generated co-culture methods that allow for in vitro recapitulation of the maternal: fetal interface. This study utilized a transwell assay, which is a co-culture model utilizing human placental syncytiotrophoblasts, fetal umbilical cells, and a differentiating embryoid body to replicate the maternal: fetal axis. To determine if cross reactive YFV vaccine antibodies impact the pathogenesis of ZIKV across the maternal fetal axis, maternal syncytiotrophoblasts were inoculated with ZIKV or ZIKV incubated with YFV vaccine anti-sera, and viral load was measured 72 hours post inoculation. Here we report that BeWo and HUVEC cells are permissive to ZIKV and that the impact of YFV post-vaccination antibodies on ZIKV replication is cell line dependent. Embryoid bodies are also permissive to ZIKV and the presence of YFV antibodies collected 1 to 6 months post vaccination enhances ZIKV infection. Our data show that each of the cell lines and EBs have a unique response to ZIKV complexed with post-vaccination serum suggesting there may be cellspecific mechanisms that impact congenital ZIKV infections. Since ZIKV infections can cause severe congenital syndromes, it is crucial to understand any potential enhancement or protection offered from cross-reactive, post-vaccination antibodies.
\end{abstract}

Keywords: Zika virus; yellow fever virus; flavivirus; cross-reactivity; neutralization; enhancement; congenital infections, Zika congenital syndrome, stem cell

\section{Introduction}

Zika Virus (ZIKV) and Yellow Fever Virus (YFV) are both part of the flavivirus family, with an enveloped, single-stranded positive-sense RNA genome. Both ZIKV and YFV are vectored by Aedes mosquitoes. Both YFV and ZIKV originated in Africa and have been found to co-circulate within the same regions of Latin America [1].. ZIKV first appeared in the Western Hemisphere in 2015 [2,3]. YFV however, has been circulating in the Americas since the African slave trade era and is endemic in many tropical regions such as Brazil, Columbia, Venezuela, and Peru to name a few [4]. In the 1930s, a live attenuated vaccine for YFV, 17D, was developed, and in its almost 80 years of use, has proven to have a significant impact on controlling YFV outbreaks [4,5]. Multiple countries have mass vaccination programs and some countries, where YFV is endemic, have the YFV-17D vaccine included in the national recommended childhood immunization schedule. Particularly, Bolivia, Brazil, Columbia, Ecuador and Venezuela all recommend the vaccine to children 9-12 months of age within the entire country, not just in known endemic regions [6]. Despite these recommendations, 
recent surveys showed that little more than half of the population in these regions are vaccinated for YFV [7]. With ongoing vaccination campaigns in these areas, there are a spectrum of post-vaccination YFV antibodies, some which might enhance infection of other flaviviruses.

With many flaviviruses co-circulating in the same areas in Central and Southern America, there is the possibility of antigenic cross reactivity, especially since some YFV endemic areas have reported seroprevalence rates of ZIKV as high as $63 \%[4,8]$. Antigenic cross reactivity and antibody mediated enhancement frequently occurs between flaviviruses. Cross-reactive Dengue virus (DENV) and West Nile virus antibodies have already been known to enhance ZIKV pathogenesis [9-11]. However, only limited studies have been conducted on the potential cross-reactive nature of YFV antibodies. One study, using commercial ELISA detection kits for DENV and ZIKV, found there to be minimal cross reactivity between YFV antibodies and DENV detection, and no cross-reactivity in ZIKV detection $[12,13]$. While these studies were very informative, they do not represent the actual immunological landscape as Souza et al [12] used post vaccination serum from 9-month old infants which have an undeveloped immune system, and the CDC MAC-ELISA for ZIKV was validated using a sample size of less than 10 with individuals of an unknown exposure history [13]. Further, South America, especially Brazil, has a high incidence of measles which can affect immunological memory in recovered persons [14].

This, however, does not indicate possible in vivo interactions as several reports indicate that flaviviral neutralization is complex and dependent upon many factors [15]. It has also been shown that antibodies that neutralize in vitro, like with neutralization assays, often do not neutralize in vivo suggesting that complex immunological interactions occur for neutralization [16-18]. In regions where ZIKV has a high prevalence, a large portion of the population also has YFV antibodies, not only from the childhood schedule of immunizations but also from ongoing vaccination campaigns that inoculate adults and provide boosters for pregnant women, HIV-infected persons, and other immunocompromised populations [19]. With a spectrum of YFV antibodies present in this population, it is important to understand any possible cross-reactivity, antibody mediated enhancement, or antibody mediated neutralization possible.

Studies have reported that vaccination of pregnant women with YFV occurs during vaccination campaigns $[20,21]$. While several studies have shown vaccination with YFV during pregnancy is safe, the development of protective immunity is reduced indicating that there may be increased nonneutralizing, cross-reactive antibodies [19-21]. Whether YFV vaccination occurs in childhood, adulthood, or during pregnancy, cross-reactive antibodies that complex with other flaviviruses could be a source for enhancement of infection. Further, non-neutralizing antibodies have been shown to contribute significantly to antibody dependent enhancement [22-24].

Since ZIKV infections can cause severe congenital syndromes, it is crucial to understand any potential enhancement or protection offered from cross-reactive antibodies [8]. Studying the vertical transmission of ZIKV has posed some challenges to researchers. Results produced in mice models are difficult to translate directly to a human or non-human primate model since mice placentas are structurally different [25-27]. Ovine and non-human primate models have proved to be promising, but these too have their limitations, such as increased costs, small sample sizes, and being labor intensive $[28,29]$. To address these roadblocks, recent advances in biotechnology have generated coculture models that use primary human cell lines and stem cells to replicate cellular interfaces. Coculture models have been used to simulate the blood brain barrier, the pulmonary barrier, and the maternal fetal axis in nanoparticle translocation studies [30-34].

The transwell co-culture model was utilized in this study to determine if the cross reactivity of YFV antibodies could impact ZIKV pathogenesis in utero during early pregnancy. This in vitro model offers multiple benefits, such as reproducibility, standardization, and excels in simulating the physiological boundary of the maternal fetal axis [35,36]. Maternal syncytiotrophoblasts and fetal 
umbilical vein cells (BeWo and HUVEC) were used in our transwell co-culture, following established placental models $[34,35,37,38]$. The BeWo cell line was derived from a human placenta and best simulates the structure and function of the syncytiotrophoblasts layer of the placenta that forms the continuous outer layer to the placenta $[39,40]$. These cells directly contact maternal blood and regulate the exchange of nutrients and particles to a developing fetus [25,41,42]. Any virus or antibodies moving across the placental barrier would first have to cross the syncytiotrophoblast layer to reach a fetus, and previous studies have determined translocation rates across a BeWo layer to replicate the rates found in ex vivo placental perfusions [27,43]. Further, we employed a ZIKV isolate derived from human placenta which we felt would be more relevant than utilizing a lab adapted strain with unknown tissue tropism in humans.

We followed Campagnolo et al. (2018) by including a differentiating embryoid body (EB) in the basolateral chamber of our transwell co-culture which mimics an early stage developing embryo [38]. An EB is generated by inducing stem cells to differentiate and self-organize in the three germ layers, endoderm, mesoderm, and ectoderm [44-46]. By including an EB in the co-culture model, we hoped to determine if there were differences in the translocation of virus and/or virus: antibody complexes that cross the placental barrier and infect an EB. Here we describe a co-culture model (Figure 1) which can be utilized to study enhancement or neutralization of virus by maternal antibodies at the maternal fetal axis. We show that YFV post-vaccine antibodies can enhance ZIKV infection of an EB which could impact the development of congenital syndromes.

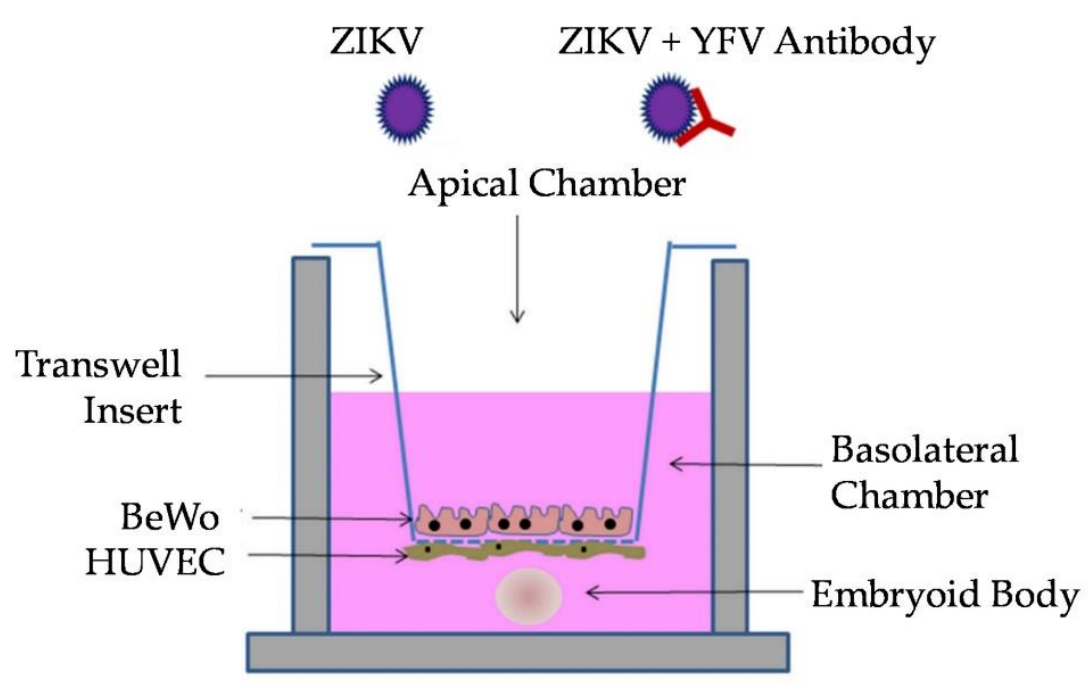

Figure 1. Experimental model of the trans-well co-culture assay modified from Campagnolo et al. (2018) [38]. Co-cultures of BeWo, HUVEC, and embryoid body (EB) were apically infected with either Zika virus (ZIKV) or ZIKV+YFV (Yellow Fever Virus) antibody.

\section{Materials and Methods}

\subsection{Cell Culture and Virus Propagation}

Primary Human Umbilical Vein Endothelial Cells; Normal, Human, Pooled (HUVEC) (ATCC PCS100-013) were cultured in EndoGRO-MV-VEGF media (MilliporeSigma, Burlington, MA, USA) containing $5 \%$ fetal bovine serum (FBS). To promote microvasculature phenotypes commonly expressed in the first trimester with placental expansion and throughout pregnancy, a variety of factors were used including rh VEGF, rh EGF, rh FGF, rh IGF, ascorbic acid, hydrocortisone hemisuccinate, heparin sulfate, and 1X Glutamax per manufacturer's instructions $[47,48]$. Additionally, human placental cells BeWo (ATCC CCL-98) were cultured in Ham's F-12K (Kaighn's) Medium containing 10\% FBS, 1X non-essential amino acids, 1X Glutamax, and 1mM HEPES. Lastly, 
Cercopithecus aethiops kidney cell line Vero E6 (ATCC CRL-1586) were grown in Dulbecco's modified Eagle's medium (DMEM) with 10\% FBS, supplemented with penicillin/streptomycin, 1X nonessential amino acids, 1X Glutamax, and 1mM HEPES. All cell lines were incubated at $37^{\circ} \mathrm{C} / 5 \% \mathrm{CO}_{2}$. ZIKV R103451 was obtained from BEI Resources (Cat. \# NR-50355) and expanded once in Vero cells. All Yellow Fever Antiserums were obtained through BEI Resources, (BEIresources.org). Pre-Immune Antiserum (Cat \#NR-41782) was taken from a non-human primate prior to immunization, Early Immune Yellow Fever Virus Antiserum (Cat \#NR-29335) was collected from a non-human primate 30 days post inoculation with live attenuated yellow fever virus vaccine (17D), Late-Immune Yellow Fever Virus Antiserum (Cat \#NR-42576) was collected from non-human primate at 30 day intervals between 120-420 days post inoculation with live attenuated yellow fever virus vaccine (17D) and then pooled, and lastly, Very Late-Immune Yellow Fever Virus Antiserum (Cat \#NR-43206) was comprised of pooled serum from non-human primate 420-570 days post inoculation with live attenuated yellow fever virus vaccine (17D).

\subsection{Embryoid Body Formation and Imaging}

Human Induced Pluripotent Stem Cells (ATCC ACS-1019) were cultured in mTeSR1 media (StemCell Technologies, Vancouver Canada) on plates coated with vitronectin XF (Stemcell Technologies, Vancouver, Canada). $100 \mu \mathrm{L}$ of undifferentiated ACS-1019 cells were seeded in each well of a 96-well round bottom ultra-low attachment plate at a density of 90,000 cells/mL using a multichannel pipettor to ensure uniformity and reproducibility of Embryoid Body (EB) formation. EB formation media

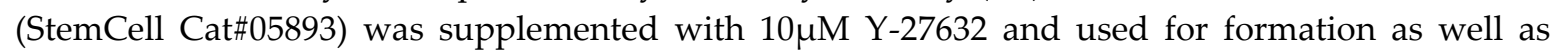
culture during the transwell assay. The 96 -well plate was incubated at $37^{\circ} \mathrm{C} / 5 \% \mathrm{CO}_{2}$ for $48 \mathrm{~h}$ without being disturbed. On day two and day four of formation, 100 $\mu \mathrm{L}$ of EB formation medium was gently added to each well. On day five, EBs were observed for uniformity, each with a diameter between $400-600 \mu \mathrm{m}$, and smooth round edges prior to being harvested and proceeding to the transwell assay.

In order to visualize ZIKV infection of EBs, EBs were rinsed in PBS, fixed with Paraformaldehyde Solution $4 \%$ in phosphate buffered saline (PBS) (ThermoScientific CAT\# J19943-K2) and blocked in $5 \%$ lamb serum. Primary antibody staining using microtubule-associated protein 2 (MAP2) (Novus Biologicals, Littleton, CO, USA) to visualize cell structure and Monoclonal Anti-Flavivirus Group Antigen, Clone D1-4G2-4-15 (BEIresources NR-50327) was conducted overnight at $4^{\circ} \mathrm{C}$. EBs were rinsed in PBS then incubated with secondary antibodies at room temperature for 1 hour. Secondary antibodies included AlexaFluor 647 and Alexafluor 488 (Invitrogen \#A21235 and A11001). EBs were rinsed again and then placed on slides and mounted in ProLong Gold Antifade Reagent with DAPI (Cell Signaling Technology, Danvers, MA, USA catalog \#8961S). Cover slips were placed on the slides and then gently pressed down to flatten the EB. Slides were incubated overnight at $4^{\circ} \mathrm{C}$. Images were taken with an Olympus Fluoview 3000 confocal microscope and processed using the Olympus Fluoview FV10-ASW 4.1 software package. All images were obtained on the same day using the same imaging parameters (zoom, gain, offset, slices, etc).

\subsection{Monolayer Infection and Imaging}

Prior to proceeding with the co-culture assay, we verified the permissiveness of BeWo and HUVEC cells to ZIKV as no reports were available to document the susceptibility of these cell lines to flaviviral infection. BeWo and HUVEC monolayers were infected with 1000 infectious units per well. After 48 hrs, samples were fixed with Paraformaldehyde Solution $4 \%$ in phosphate buffered saline (PBS) (ThermoScientific CAT\# J19943-K2) and blocked in 5\% lamb serum. Primary antibody staining with microtubule-associated protein 2 (MAP2) (Novus Biologicals, Littleton, CO, USA) was used to visualize cell structure and anti-flavivirus group antigen, Clone D1-4G2-4-15 4G2 (BEIresources NR50327) to visualize ZIKV. Staining was conducted overnight at $4^{\circ} \mathrm{C}$. Cells were rinsed in PBS then incubated with secondary antibodies at room temperature for 1 hour. Secondary antibodies included AlexaFluor 647 and Alexafluor 488 (Invitrogen \#A21235 and A11001). Cells were rinsed and then coverslips were mounted with ProLong Gold Antifade Reagent with DAPI (Cell Signaling Technology, Danvers, MA, USA catalog \#8961S) and incubated overnight at $4^{\circ} \mathrm{C}$. Images were taken 
with an Olympus Fluoview 3000 confocal microscope and processed using the Olympus Fluoview FV10-ASW 4.1 software package.

\subsection{Transwell Co-Culture}

We followed methods previously described elsewhere [49,50]. Briefly, HUVEC cells were seeded on the basolateral side of the Corning $12 \mathrm{~mm}$ Trans-well@-COL Collagen-Coated $3.0 \mu \mathrm{m}$ Pore PTFE Membrane Insert (Corning, NY, USA catalog\# 3494) at a concentration of $1.0 \times 10^{5}$ cells per $200 \mu 1$. The HUVEC monolayer on the basolateral side was achieved using methods described by Aengenheister et al. (2018) [35]. Briefly, inserts were inverted into 6 well plates, with $1 \mathrm{~mL}$ of PBS in one well to ensure enough humidity. Rubber spacers (approximately $1.5 \mathrm{~mm}$ thick) were placed on the corner of the 6 well plate to lift the lid slightly and prevent direct contact of the lid with the inverted insert. After the basolateral side was seeded with HUVECs and the lid was replaced, there was slight adhesion between the lid and the media. HUVEC seeded inserts were then incubated at $37^{\circ} \mathrm{C} / 5 \%$ $\mathrm{CO} 2$ for $2 \mathrm{hrs}$, and afterwards the inserts were placed back into the 12 well plate containing fresh HUVEC Media. After an insert was replaced in the 12 well plate the apical layer of the membrane was seeded with BeWo cells at a density of $1.5 \times 105$ cells per $500 \mu 1$. Co-cultures were incubated for $72 \mathrm{hrs}$ with the media being changed every $48 \mathrm{hrs}$ until a $100 \%$ confluent layer was observed.

\subsection{Transwell Neutralization Assay}

Prior to infection, the media in each basolateral chamber was replaced with 1/2 HUVEC media 1/2 EB Formation media. Three EBs were added to the bottom of each well (figure 1). Virus assays using YFV serum were performed using a 1:200 dilution of serum in PBS. 1,000 infectious units of virus in PBS were incubated with serum for 1 hour at $37^{\circ} \mathrm{C}$ after which BeWo cells were inoculated with the mixture in the apical chamber. Assay controls included treatments of mock infection with PBS, PreImmune Antiserum, Early-Immune Antiserum, Late-Immune Antiserum, Very Late-Immune Antiserum, and virus only. To obtain neutralization data, supernatant from BeWo and HUVEC cells was taken at $72 \mathrm{hrs}$ post inoculation and all 3 replicates per cell line were pooled to obtain a single combined solution which was titrated on Vero E6 cells (Figure 2). EBs were separated from the HUVEC supernatant by centrifugation at 400xg for 5 minutes. The supernatant was aspirated and EBs were washed with PBS before a second centrifugation at $400 \mathrm{xg}$ for 5 minutes, after which they were gently resuspended in PBS. 3 EBs from each replicate were pooled for a total of 9 EBs per treatment for each independent trial. Results of the titration assay are expressed as an average between two independent trials with three replicates for each treatment. A Student's t-test was used for pairwise comparisons between the treatments. All virus:antibody assays were compared against the ZIKV only assay.

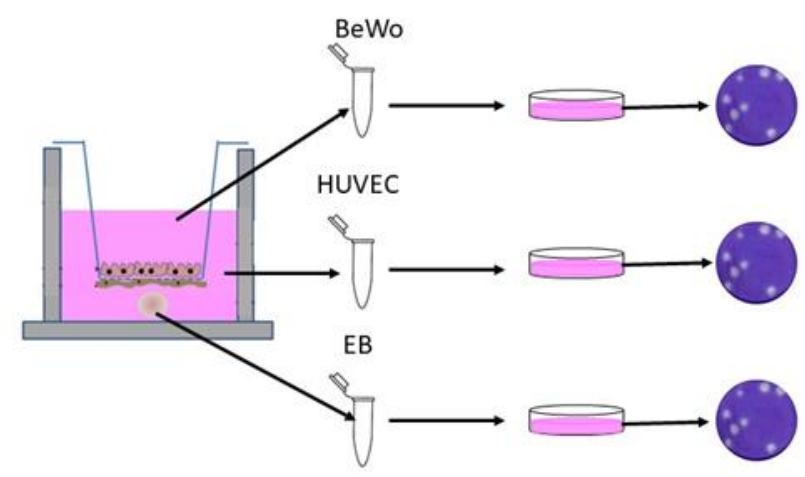

Figure 2. Transwell neutralization assay. Supernatant from BeWo and HUVEC cells was taken at 72 hrs post inoculation and all 3 replicates per cell line were pooled to obtain a single combined solution which was titrated on Vero E6 cells. EBs were separated from the HUVEC media and washed in PBS. 3 EBs from each replicate were pooled for a total of 9 EBs per treatment for each independent trial. This figure was modified based on the original illustration by Campagnolo et al. 2018 [38]. 
Plaque assays were performed using pooled supernatant samples from each treatment following a method described previously [51]. EBs were separated as described above, and vigorously triturated to disassociate cells. Briefly, serial dilutions of culture supernatant or EBs in PBS were inoculated onto confluent Vero E6 cells and covered with $0.25 \%$ methylcellulose overlay. Overlay was removed after three days and cells were fixed and stained with 5\% acetic acid, 43\% ethanol, 50\% methanol and $0.2 \% \mathrm{wt} / \mathrm{vol}$ Coomassie Brilliant Blue R-250 prior to counting plaques. Pairwise comparisons between virus:antibody treatments and ZIKV only were performed using a Student's t-test.

\section{Results}

3.1. Maternal and Fetal Placental Cells are Permissive to ZIKV Infection

A
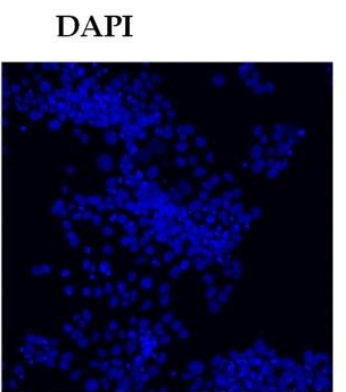

BeWo

Control

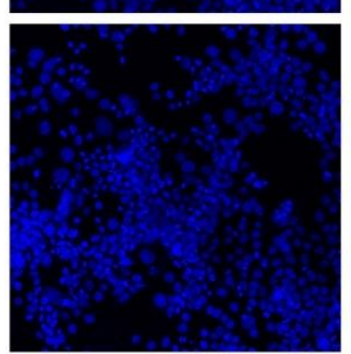

B

DAPI

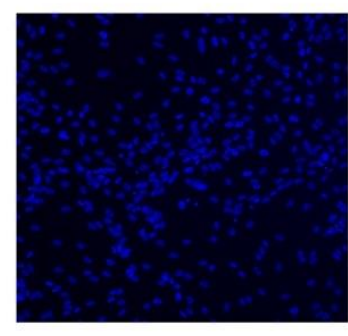

HUVEC

Control

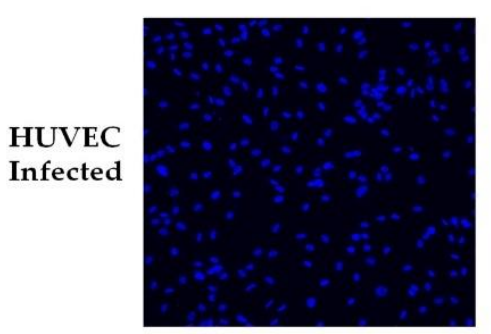

MAP2
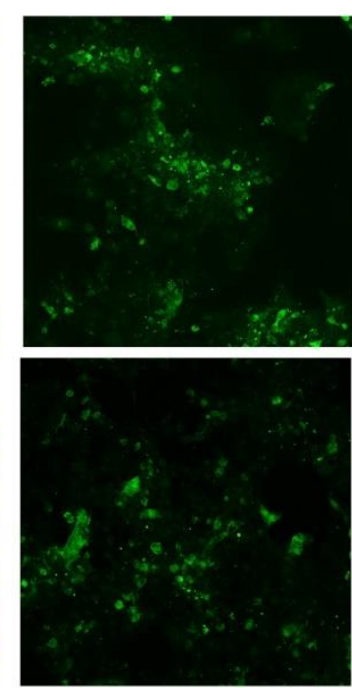

MAP2
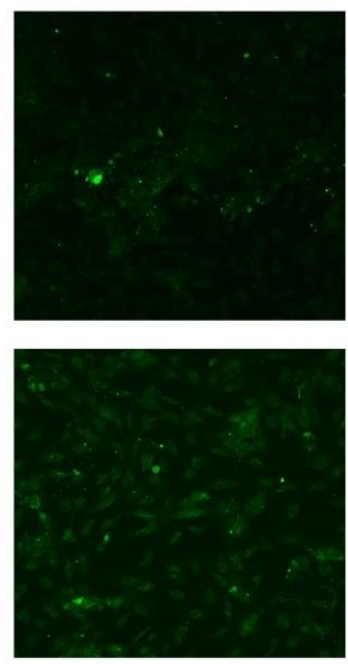

ZIKV
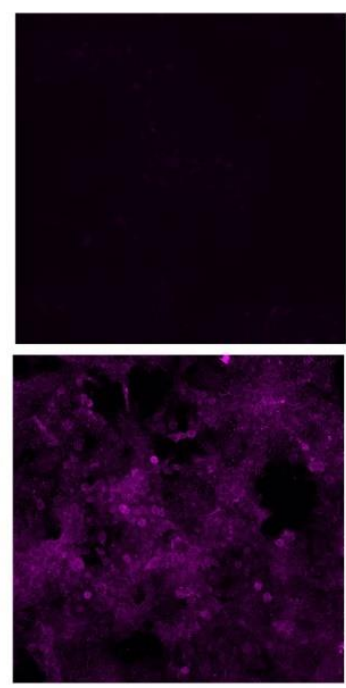

ZIKV
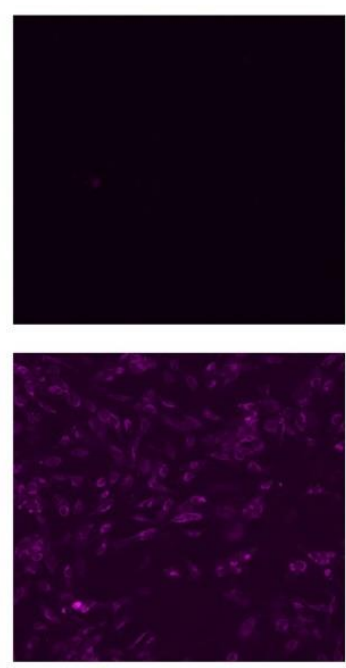

Merge
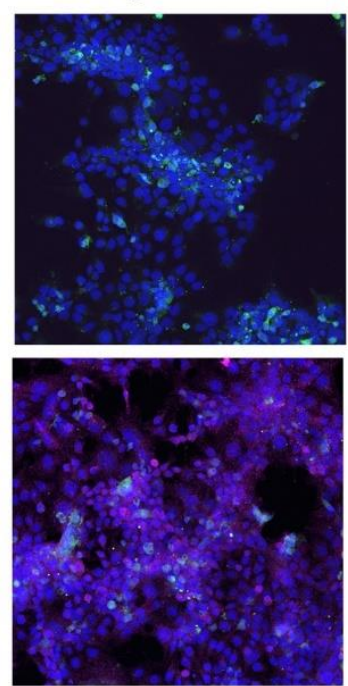

Merge
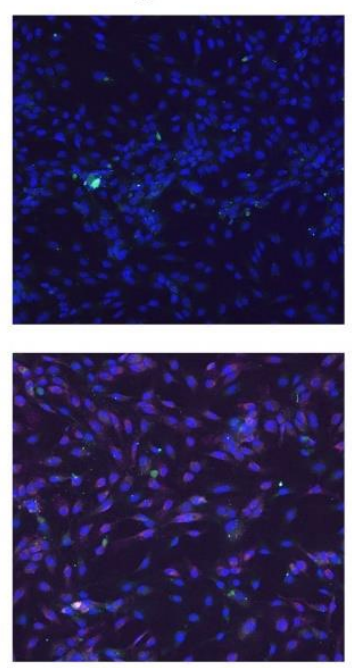

Figure 3. Monolayer infection of BeWo and HUVEC cells with ZIKV viewed under 20x magnification. (A) BeWo monolayer stained $48 \mathrm{hrs}$ post inoculation with ZIKV. (B) HUVEC monolayer stained $48 \mathrm{hrs}$ post inoculation. (Blue=DAPI, Green=MAP2, Pink= ZIKV: 4G2).

Clinical reports indicate that placental pathology is minimal for ZIKV congenital infections and primarily involves reduced placental weight [52-54]. Reports have shown that ZIKV causes autophagy, apoptosis and other forms of CPE in HUVEC cells which is contrary to clinical data [55,56]. ZIKV has been detected in BeWo 
cells also but investigations regarding CPE was limited [57,58]. Further these studies did not employ the placental isolate used here which was derived from the placenta of an infant with microcephaly which could impact observations [59]. The purpose of this experiment was to determine if our ZIKV strain was permissive to HUVEC and BeWo cells and that it would not induce excessive cytopathic effects that might interfere with the experiment. Monolayers of both BeWo and HUVEC cell lines were infected with ZIKV and infection visualized using the anti-ZIKV D1-4G2-4-15 antibody. Staining determined that both BeWo and HUVEC cell lines are permissive to ZIKV infection (Figure 3). This aligns with previous research showing the placental trophoblasts and endothelial cells to be permissive to ZIKV infection [60-63] There were no noticeable cytopathic effects within BeWo and HUVEC cell lines when infected with ZIKV (Figure 3).

\subsection{Transwell Neutralization Assay}

At $72 \mathrm{hrs}$ post inoculation (p.i.), ZIKV was detected via viral plaque assay. Very Late Antiserum significantly increased the amount of infectious ZIKV present in BeWo cells at 72hrs post inoculation as compared to when no Anti-serum was present in the transwell assay $(\mathrm{p}=0.033)$ (Table 1, Figure 4). The presence of YFV antibodies, regardless of time the antiserum was taken post vaccination, did not have any significant impact on ZIKV viral titers or Ct-values in the HUVEC cell line (Table 1). EBs had significantly increased levels of infectious ZIKV at $72 \mathrm{hrs}$ p.i. when Early and Late YFV Anti-serum were used as when compared to no anti-serum present ( $\mathrm{p}=0.0294$, $\mathrm{p}=0.000762$ ) (Table 1). Imaging studies of the EB verified that ZIKV could be detected throughout the EB and $\mathrm{CPE}$ was inapparent in all treatments (Figure 4). The variation in size observed reflects that normal variance of size when EBs are generated in the manner we employed [64].

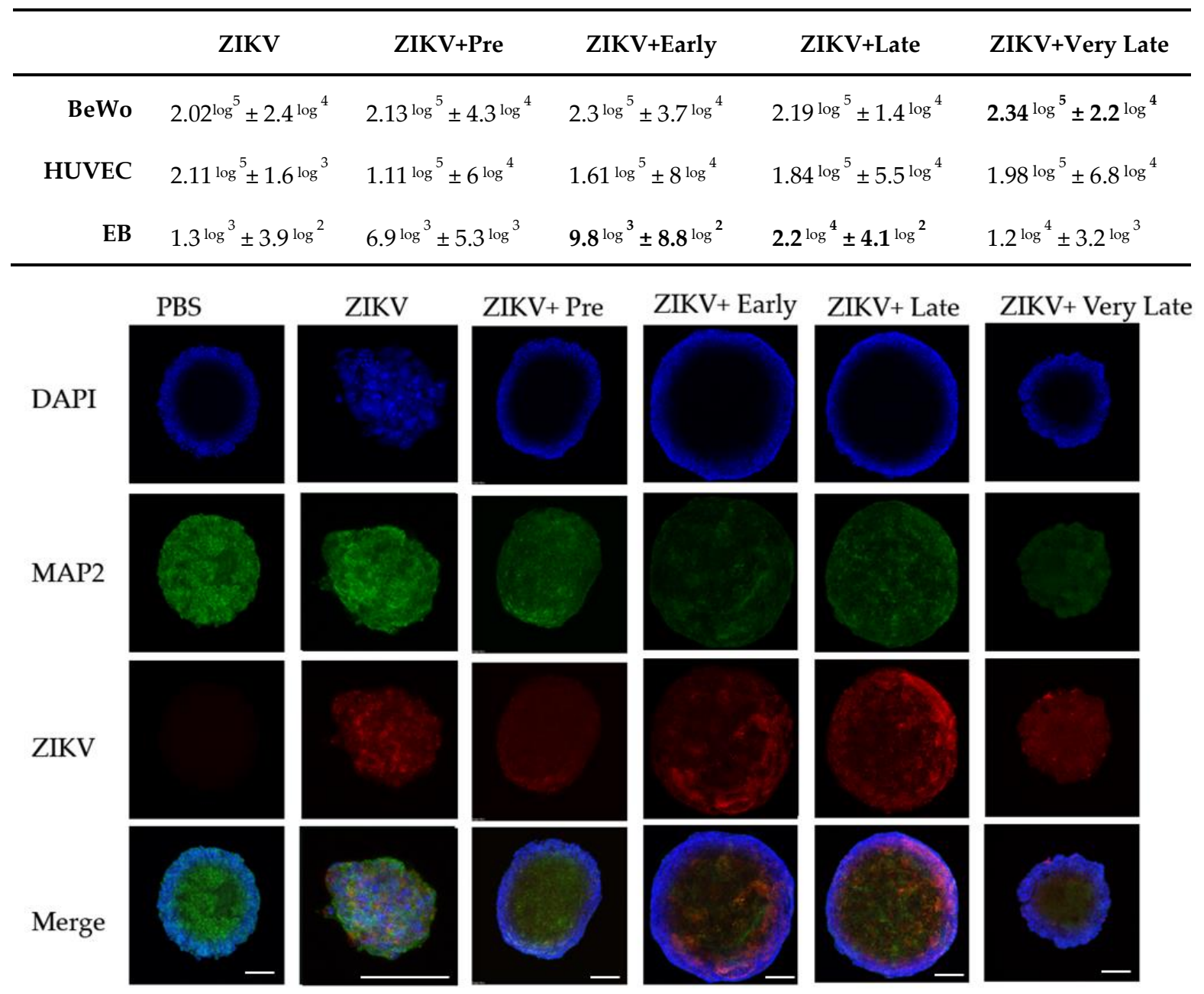

Table 1. Viral titration of ZIKV in BeWo, HUVEC, and EBs $72 \mathrm{hr}$ p.i. Data provided signifies average ${ }^{\log }$ titer \pm standard error of 2 independent trials with 3 replicates each. Significant increases in ZIKV titers were seen in BeWo cells when Very Late YFV anti-serum was present when compared to ZIKV only ( $\mathrm{p}=0.0334$ ), and in EBs 
when Early $(p=0.0294)$ and Late $(p=0.000762)$ YFV anti-serum was present when compared to ZIKV only. Bold font indicates statistical significance.

Figure 4. Immunofluorescence of EBs. (Blue=DAPI, Green=MAP2, Red= ZIKV: 4G2). EBs were stained with D14G2-4-15 antibody and mounted on glass slides. Coverslips were pressed down on the EBs to flatten them, producing a ring effect in most images. Scale bar represents 100uM.

\section{Discussion}

Here, we present data showing that ZIKV derived from the human placenta can infect both BeWo and HUVEC cells and replicate without out causing excessive CPE. ZIKV has been known to infect placental tissue in humans as well as in animal models, and it can cross the placental barrier to infect a developing fetus to cause congenital syndromes [11,28,65-67]. The data in this study shows that within $72 \mathrm{hrs}$ p.i., ZIKV effectively crosses two monolayers, a basement membrane of placental collagen, and infects an EB. The detection of ZIKV in cells located on each side of the membrane as a model for the maternal: fetal axis supports reports of isolation of ZIKV from placentas and fetuses $[28,53,68,69]$. However, the permissiveness of cells observed in this study may not reflect the cellular tropism of ZIKV in actual placentas [70-73].

Pre-Vaccination sera was collected prior to vaccination with the 17D YFV vaccine, Early serum was collected 30 days p.i., Late serum was collected at 30 days intervals between 120-420 days p.i., and lastly, Very Late serum was comprised of pooled serum from 420-570 days p.i.. It has been shown that broadly neutralizing IgM antibodies quickly appear after vaccination with the 17D vaccine, typically 4-7 days p.i., and have been found to circulate anywhere between 2-11 years p.i. [74,75]. IgM antibodies are broadly reactive while IgG antibodies are associated with a higher antigenic specificity and better immune responses. IgG antibodies take longer to appear after vaccination, usually 28-42 days p.i. with the 17D vaccine, IgM is present in significantly higher titers than IgG [75]. This could explain why there was a significant enhancement of ZIKV infection in EBs when Early and LateImmune YFV-Antiserum were present. These differences are important to consider when analyzing why some sera in this study enhanced while others did not. IgG antibodies have Fc regions and can readily cross the placenta due the $\mathrm{FcRn}$ receptor on placental syncytiotrophoblasts and endothelial cells and have already been found to be endocytosed by the BeWo cell line [76-79]. By the time Early and Late antiserum was collected, both IgM and IgG antibodies would have been abundant, suggesting why ZIKV enhancement was observed in BeWo and EBs.

Anti-DENV antibodies have been shown to enhance ZIKV infection in multiple model systems and case studies [9,11,79-81]. Due to the genetic similarities between flaviviruses it would be expected that antibodies for other flaviviruses, such as YFV, may also contain the ability to enhance ZIKV infection. In this study, EBs had enhanced ZIKV infection along with lower viral loads as compared to the placental cell lines. This supports reports of ZIKV titers in fetal and placental tissues in vivo $[28,68,69]$. Since macrophages or other monocytes were not used in this study, the enhancement we observed was likely due to antibody mediated enhancement (AME). Like ADE, AME occurs when antibodies bind to virus particles forming complexes. These complexes interact with cell surface receptors and promote entry into host cells leading to increased levels of viral replication via suppression of innate immune processes and inflammatory cascades [23,82]. While this process is associated with Fc receptor-bearing monocytes, it is also possible for these virus-antibody complexes to infect other cell types and suppress innate immunity $[23,82]$.

A limitation to this study was the omission of Hofbauer cells from the model. Hofbauer cells have been found to play a role in ZIKV transmission through the placental barrier [79,83-85]. They are placental macrophages that also have Fc receptors, play a role in early angiogenesis within trophoblast cells, and have been found to transfer viral particles into the fetal endothelial cells and blood supply [77,86-88]. Since they have been found to further facilitate vertical transmission of ZIKV, 
not including them in the transwell co-culture assay limits the findings within this study. DENV antibodies have already been found to enhance ZIKV infection within Hofbauer cells, and as such not including them could have impacted the results [79].

\section{Conclusions}

Due to the already established cross reactive nature of flavivirus antibodies, it is crucial to understand the interactions of neutralizing and enhancing antibodies as vaccine research continues for many of these viruses $[9,10,89,90]$. The 17D vaccine for YFV has been included in the recommended vaccinations in most Latin American countries and ongoing vaccination campaigns are vaccinating more adults, creating a population of people with potentially cross-reactive antibodies $[4-6,12,75]$. Additionally, in regions of Latin America where these viruses co-circulate due to a common vector, Aedes mosquitos, many people have the potential to become co-infected or infected by different flaviviruses within their lifetime $[1,4,8,84]$. Further research about the cross-reactive nature of flavivirus antibodies is needed, especially because vertically transmitted viral infections can lead to congenital syndromes. More studies are also needed to better understand the kinetics of antibody passage through the transwell co-culture model, and to better understand possible antibody mediated enhancement of ZIKV by YFV antibodies produced after receiving the 17D vaccine especially in pregnant women who may be receiving a booster or receiving vaccination during campaigns.

Author Contributions: Conceptualization, KLB and EMS.; methodology, KLB, EMS, TJJ; validation, KLB and EMS formal analysis, KLB and EMS.; investigation, KLB, EMS, TJJ.; writing - original draft preparation, KLB and EMS.; writing - review and editing, KLB, EMS, TJJ. All authors have read and agreed to the published version of the manuscript.

Funding: This research received no external funding.

Acknowledgments: This work was supported by start-up funds to Dr. Kelli Barr from Baylor University. We thank Dr. Campagnolo for allowing us to utilize his figure in this manuscript.

Conflicts of Interest: The authors declare no conflict of interest. The funders had no role in the design of the study; in the collection, analyses, or interpretation of data; in the writing of the manuscript, or in the decision to publish the results.

\section{References}

1. Charlier, C.; Beaudoin, M.-C.; Couderc, T.; Lortholary, O.; Lecuit, M. Arboviruses and pregnancy: maternal, fetal, and neonatal effects. The Lancet Child $\mathcal{E}$ Adolescent Health 2017, 1, 134-146, doi:10.1016/s2352-4642(17)30021-4.

2. Haddow, A.J.; Williams, M.C.; Woodall, J.P.; Simpson, D.I.; Goma, L.K. TWELVE ISOLATIONS OF ZIKA VIRUS FROM AEDES (STEGOMYIA) AFRICANUS (THEOBALD) TAKEN IN AND ABOVE A UGANDA FOREST. Bull World Health Organ 1964, 31, 57-69.

3. Zanluca, C.; Melo, V.C.A.d.; Mosimann, A.L.P.; Santos, G.I.V.d.; Santos, C.N.D.d.; Luz, K. First report of autochthonous transmission of Zika virus in Brazil. Memórias do Instituto Oswaldo Cruz 2015, 110, 569572.

4. Douam, F.; Ploss, A. Yellow Fever Virus: Knowledge Gaps Impeding the Fight Against an Old Foe. Trends in Microbiology 2018, 26, 913-928, doi:https://doi.org/10.1016/j.tim.2018.05.012.

5. Barrett, A.D.T. Yellow fever live attenuated vaccine: A very successful live attenuated vaccine but still we have problems controlling the disease. Vaccine 2017, 35, 5951-5955, doi:https://doi.org/10.1016/j.vaccine.2017.03.032.

6. WHO. WHO vaccine-preventable diseases: monitoring system. Availabe online: (accessed on 
7. WHO. Yellow Fever- Brazil. Availabe online: https://www.who.int/csr/don/18-april-2019-yellow-feverbrazil/en/ (accessed on

8. Netto, E.M.; Moreira-Soto, A.; Pedroso, C.; Höser, C.; Funk, S.; Kucharski, A.J.; Rockstroh, A.; Kümmerer, B.M.; Sampaio, G.S.; Luz, E., et al. High Zika Virus Seroprevalence in Salvador, Northeastern Brazil Limits the Potential for Further Outbreaks. mBio 2017, 8, e01390-01317, doi:10.1128/mBio.01390-17.

9. Wen, J.; Shresta, S. Antigenic cross-reactivity between Zika and dengue viruses: is it time to develop a universal vaccine? Current Opinion in Immunology 2019, 59, 1-8, doi:https://doi.org/10.1016/j.coi.2019.02.001.

10. Willis, E.; Hensley, S.E. Characterization of Zika virus binding and enhancement potential of a large panel of flavivirus murine monoclonal antibodies. Virology 2017, 508, 1-6, doi:https://doi.org/10.1016/j.virol.2017.04.031.

11. Hermanns, K.; Göhner, C.; Kopp, A.; Schmidt, A.; Merz, W.M.; Markert, U.R.; Junglen, S.; Drosten, C. Zika virus infection in human placental tissue explants is enhanced in the presence of dengue virus antibodies in-vitro. Emerg Microbes Infect 2018, 7, 198-198, doi:10.1038/s41426-018-0199-6.

12. Souza, N.C.S.e.; Félix, A.C.; de Paula, A.V.; Levi, J.E.; Pannuti, C.S.; Romano, C.M. Evaluation of serological cross-reactivity between yellow fever and other flaviviruses. International Journal of Infectious Diseases 2019, 81, 4-5, doi:https://doi.org/10.1016/j.ijid.2019.01.023.

13. CDC. Zika MAC-ELISA: Instructions for Use; 2018.

14. WHO. Global Measles and Rubella Update May 2020. Availabe online: https://www.who.int/immunization/monitoring surveillance/burden/vpd/surveillance type/active/m easles_monthlydata/en/ (accessed on

15. Pierson, T.C.; Diamond, M.S. Molecular mechanisms of antibody-mediated neutralisation of flavivirus infection. Expert Rev Mol Med 2008, 10, e12, doi:10.1017/s1462399408000665.

16. Throsby, M.; Geuijen, C.; Goudsmit, J.; Bakker, A.Q.; Korimbocus, J.; Kramer, R.A.; Clijsters-van der Horst, M.; de Jong, M.; Jongeneelen, M.; Thijsse, S., et al. Isolation and Characterization of Human Monoclonal Antibodies from Individuals Infected with West Nile Virus. Journal of Virology 2006, 80, 6982, doi:10.1128/JVI.00551-06.

17. Schlesinger, J.J.; Chapman, S. Neutralizing F(ab')2 fragments of protective monoclonal antibodies to yellow fever virus (YF) envelope protein fail to protect mice against lethal YF encephalitis. J Gen Virol 1995, 76 ( Pt 1), 217-220, doi:10.1099/0022-1317-76-1-217.

18. Oliphant, T.; Nybakken, G.E.; Engle, M.; Xu, Q.; Nelson, C.A.; Sukupolvi-Petty, S.; Marri, A.; Lachmi, B.E.; Olshevsky, U.; Fremont, D.H., et al. Antibody recognition and neutralization determinants on domains I and II of West Nile Virus envelope protein. J Virol 2006, 80, 12149-12159, doi:10.1128/jvi.01732-06.

19. Fischer, J.E.S.J.A.B.J.L.R.M. Yellow Fever Vaccine Booster Doses: Recommendations of the Advisory Committee on Immunization Practices, 2015. Availabe online: https://www.cdc.gov/mmwr/preview/mmwrhtml/mm6423a5.htm (accessed on

20. Thomas, R.E.; Lorenzetti, D.L.; Spragins, W.; Jackson, D.; Williamson, T. The safety of yellow fever vaccine 17D or 17DD in children, pregnant women, HIV+ individuals, and older persons: systematic review. Am J Trop Med Hyg 2012, 86, 359-372, doi:10.4269/ajtmh.2012.11-0525. 
21. Gotuzzo, E.; Yactayo, S.; Córdova, E. Efficacy and duration of immunity after yellow fever vaccination: systematic review on the need for a booster every 10 years. Am J Trop Med Hyg 2013, 89, 434-444, doi:10.4269/ajtmh.13-0264.

22. Sanchez, M.D.; Pierson, T.C.; McAllister, D.; Hanna, S.L.; Puffer, B.A.; Valentine, L.E.; Murtadha, M.M.; Hoxie, J.A.; Doms, R.W. Characterization of neutralizing antibodies to West Nile virus. Virology 2005, 336, 70-82, doi:10.1016/j.virol.2005.02.020.

23. Takada, A.; Kawaoka, Y. Antibody-dependent enhancement of viral infection: molecular mechanisms and in vivo implications. Reviews in medical virology 2003, 13, 387-398, doi:10.1002/rmv.405 10.1002/rmv.405.

24. A, T.; S., F.S.; R, B.; V., D.L.; J., K.N.; Mahalingam, S. Fc receptors in antibody-dependent enhancement of viral infections. Immunology Reviews 2015, 268, 24, doi:10.1111/imr.12367.

25. Arora, N.; Sadovsky, Y.; Dermody, T.S.; Coyne, C.B. Microbial Vertical Transmission during Human Pregnancy. Cell Host Microbe 2017, 21, 561-567, doi:10.1016/j.chom.2017.04.007.

26. Schmidt, A.; Morales-Prieto, D.M.; Pastuschek, J.; Fröhlich, K.; Markert, U.R. Only humans have human placentas: molecular differences between mice and humans. Journal of Reproductive Immunology 2015, 108, 65-71, doi:https://doi.org/10.1016/j.jri.2015.03.001.

27. Poulsen, M.S.; Rytting, E.; Mose, T.; Knudsen, L.E. Modeling placental transport: Correlation of in vitro BeWo cell permeability and ex vivo human placental perfusion. Toxicology in Vitro 2009, 23, 1380-1386, doi:https://doi.org/10.1016/j.tiv.2009.07.028.

28. Schwarz, E.R.; Pozor, M.A.; Pu, R.; Barr, K.L.; Beachboard, S.E.; MacLachlan, N.J.; Prakoso, D.; Long, M.T. Experimental Infection of Pregnant Female Sheep with Zika Virus During Early Gestation. Viruses 2019, 11, 795, doi:10.3390/v11090795.

29. Steinbach, R.J.; Haese, N.N.; Smith, J.L.; Colgin, L.M.A.; MacAllister, R.P.; Greene, J.M.; Parkins, C.J.; Kempton, J.B.; Porsov, E.; Wang, X., et al. A neonatal nonhuman primate model of gestational Zika virus infection with evidence of microencephaly, seizures and cardiomyopathy. PLOS ONE 2020, 15, e0227676, doi:10.1371/journal.pone.0227676.

30. Appelt-Menzel, A.; Cubukova, A.; Günther, K.; Edenhofer, F.; Piontek, J.; Krause, G.; Stüber, T.; Walles, H.; Neuhaus, W.; Metzger, M. Establishment of a Human Blood-Brain Barrier Co-culture Model Mimicking the Neurovascular Unit Using Induced Pluri- and Multipotent Stem Cells. Stem Cell Reports 2017, 8, 894-906, doi:https://doi.org/10.1016/j.stemcr.2017.02.021.

31. Zhang, F.; Aquino, G.V.; Dabi, A.; Bruce, E.D. Assessing the translocation of silver nanoparticles using an in vitro co-culture model of human airway barrier. Toxicology in Vitro 2019, 56, 1-9, doi:https://doi.org/10.1016/j.tiv.2018.12.013.

32. Dekali, S.; Gamez, C.; Kortulewski, T.; Blazy, K.; Rat, P.; Lacroix, G. Assessment of an in vitro model of pulmonary barrier to study the translocation of nanoparticles. Toxicology Reports 2014, 1, 157-171, doi:https://doi.org/10.1016/j.toxrep.2014.03.003.

33. Garcia, C.M.; Darland, D.C.; Massingham, L.J.; D'Amore, P.A. Endothelial cell-astrocyte interactions and TGF $\beta$ are required for induction of blood-neural barrier properties. Developmental Brain Research 2004, 152, 25-38, doi:https://doi.org/10.1016/j.devbrainres.2004.05.008.

34. Cartwright, L.; Poulsen, M.S.; Nielsen, H.M.; Pojana, G.; Knudsen, L.E.; Saunders, M.; Rytting, E. In vitro placental model optimization for nanoparticle transport studies. Int J Nanomedicine 2012, 7, 497510, doi:10.2147/IJN.S26601. 
35. Aengenheister, L.; Keevend, K.; Muoth, C.; Schönenberger, R.; Diener, L.; Wick, P.; Buerki-Thurnherr, T. An advanced human in vitro co-culture model for translocation studies across the placental barrier. Scientific Reports 2018, 8, 5388, doi:10.1038/s41598-018-23410-6.

36. Myllynen, P.; Vähäkangas, K. Placental transfer and metabolism: An overview of the experimental models utilizing human placental tissue. Toxicology in Vitro 2013, 27, 507-512, doi:https://doi.org/10.1016/j.tiv.2012.08.027.

37. Aengenheister, L.; Dietrich, D.; Sadeghpour, A.; Manser, P.; Diener, L.; Wichser, A.; Karst, U.; Wick, P.; Buerki-Thurnherr, T. Gold nanoparticle distribution in advanced in vitro and ex vivo human placental barrier models. J Nanobiotechnology 2018, 16, 79-79, doi:10.1186/s12951-018-0406-6.

38. Campagnolo L , L.V., Massimiani M, Magrini A and Pietroiusti A. In vitro experimental models to study the efficiency of the placental barrier for environmental toxicants: tumor cell lines versus trophoblast primary cells. Biomedicine and Prevention 2018, 1, doi:10.19252/00000009D.

39. Orendi, K.; Kivity, V.; Sammar, M.; Grimpel, Y.; Gonen, R.; Meiri, H.; Lubzens, E.; Huppertz, B. Placental and trophoblastic in vitro models to study preventive and therapeutic agents for preeclampsia. Placenta 2011, 32, S49-S54, doi:https://doi.org/10.1016/j.placenta.2010.11.023.

40. Evseenko, D.A.; Paxton, J.W.; Keelan, J.A. ABC drug transporter expression and functional activity in trophoblast-like cell lines and differentiating primary trophoblast. American Journal of PhysiologyRegulatory, Integrative and Comparative Physiology 2006, 290, R1357-R1365, doi:10.1152/ajpregu.00630.2005.

41. Wang Y, Z.S. Cell Types of the Placenta. In Vascular Biology of the Placenta, Morgan \& Claypool Life Sciences: San Rafael, CA, 2010.

42. Heaton, S.J.; Eady, J.J.; Parker, M.L.; Gotts, K.L.; Dainty, J.R.; Fairweather-Tait, S.J.; McArdle, H.J.; Srai, K.S.; Elliott, R.M. The use of BeWo cells as an in vitro model for placental iron transport. Am J Physiol Cell Physiol 2008, 295, C1445-C1453, doi:10.1152/ajpcell.00286.2008.

43. Li, H.; Ravenzwaay, B.v.; Rietjens, I.M.C.M.; Louisse, J. Assessment of an in vitro transport model using BeWo b30 cells to predict placental transfer of compounds. Archives of Toxicology 2013, 87, 1661-1669, doi:10.1007/s00204-013-1074-9.

44. Itskovitz-Eldor, J.; Schuldiner, M.; Karsenti, D.; Eden, A.; Yanuka, O.; Amit, M.; Soreq, H.; Benvenisty, N. Differentiation of human embryonic stem cells into embryoid bodies compromising the three embryonic germ layers. Mol Med 2000, 6, 88-95.

45. Guo, N.-N.; Liu, L.-P.; Zhang, Y.-X.; Cai, Y.-T.; Guo, Y.; Zheng, Y.-W.; Li, Y.-M. Early prediction of the differentiation potential during the formation of human iPSC-derived embryoid bodies. Biochemical and Biophysical Research Communications 2019, 516, 673-679, doi:https://doi.org/10.1016/j.bbrc.2019.06.081.

46. Sheridan, S.D.; Surampudi, V.; Rao, R.R. Analysis of embryoid bodies derived from human induced pluripotent stem cells as a means to assess pluripotency. Stem Cells Int 2012, 2012, 738910, doi:10.1155/2012/738910.

47. Leach, L.; Badet, J.; Brownbill, P.; Harris, L.; Keogh, R.; Kalionis, B.; Whitley, G. Endothelium, Blood Vessels and Angiogenesis - A Workshop Report. Placenta 2006, 27, 26-29, doi:https://doi.org/10.1016/j.placenta.2006.01.022.

48. Lang, I.; Pabst, M.A.; Hiden, U.; Blaschitz, A.; Dohr, G.; Hahn, T.; Desoye, G. Heterogeneity of microvascular endothelial cells isolated from human term placenta and macrovascular umbilical vein endothelial cells. European Journal of Cell Biology 2003, 82, 163-173, doi:https://doi.org/10.1078/0171-933500306. 
49. Schultz, E.M.; Jones, T.J.; Barr, K.L. Antibodies for Venezuelan Equine Encephalitis Virus Protect Embryoid Bodies from Chikungunya Virus. Viruses 2020, 12, doi:10.3390/v12030262.

50. Aengenheister, L.; Keevend, K.; Muoth, C.; Schonenberger, R.; Diener, L.; Wick, P.; Buerki-Thurnherr, T. An advanced human in vitro co-culture model for translocation studies across the placental barrier. Sci Rep 2018, 8, 5388, doi:10.1038/s41598-018-23410-6 10.1038/s41598-018-23410-6.

51. Barr, K.L.; Schwarz, E.R.; Prakoso, D.; Imtiaz, K.; Pu, R.; Morris Jr, J.G., Jr.; Khan, E.; Long, M.T. StrainDependent Activity of Zika Virus and Exposure History in Serological Diagnostics. Trop Med Infect Dis 2020, 5, 38, doi:10.3390/tropicalmed5010038.

52. Mlakar, J.; Korva, M.; Tul, N.; Popović, M.; Poljšak-Prijatelj, M.; Mraz, J.; Kolenc, M.; Resman Rus, K.; Vesnaver Vipotnik, T.; Fabjan Vodušek, V., et al. Zika Virus Associated with Microcephaly. The New England journal of medicine 2016, 374, 951-958, doi:10.1056/NEJMoa1600651.

53. Reagan-Steiner, S.; Simeone, R.; Simon, E.; Bhatnagar, J.; Oduyebo, T.; Free, R.; Denison, A.M.; Rabeneck, D.B.; Ellington, S.; Petersen, E., et al. Evaluation of Placental and Fetal Tissue Specimens for Zika Virus Infection - 50 States and District of Columbia, January-December, 2016. MMWR Morb Mortal Wkly Rep 2017, 66, 636-643, doi:10.15585/mmwr.mm6624a3.

54. Martines, R.B.; Bhatnagar, J.; de Oliveira Ramos, A.M.; Davi, H.P.; Iglezias, S.D.; Kanamura, C.T.; Keating, M.K.; Hale, G.; Silva-Flannery, L.; Muehlenbachs, A., et al. Pathology of congenital Zika syndrome in Brazil: a case series. Lancet 2016, 388, 898-904, doi:10.1016/s0140-6736(16)30883-2.

55. Peng, H.; Liu, B.; Yves, T.D.; He, Y.; Wang, S.; Tang, H.; Ren, H.; Zhao, P.; Qi, Z.; Qin, Z. Zika Virus Induces Autophagy in Human Umbilical Vein Endothelial Cells. Viruses 2018, 10, doi:10.3390/v10050259.

56. Anfasa, F.; Goeijenbier, M.; Widagdo, W.; Siegers, J.Y.; Mumtaz, N.; Okba, N.; van Riel, D.; Rockx, B.; Koopmans, M.P.G.; Meijers, J.C.M., et al. Zika Virus Infection Induces Elevation of Tissue Factor Production and Apoptosis on Human Umbilical Vein Endothelial Cells. Front Microbiol 2019, 10, 817, doi:10.3389/fmicb.2019.00817.

57. Zani, A.; Zhang, L.; McMichael, T.M.; Kenney, A.D.; Chemudupati, M.; Kwiek, J.J.; Liu, S.L.; Yount, J.S. Interferon-induced transmembrane proteins inhibit cell fusion mediated by trophoblast syncytins. The Journal of biological chemistry 2019, 294, 19844-19851, doi:10.1074/jbc.AC119.010611.

58. Bayer, A.; Lennemann, N.J.; Ouyang, Y.; Bramley, J.C.; Morosky, S.; Marques, E.T.; Cherry, S.; Sadovsky, Y.; Coyne, C.B. Type III Interferons Produced by Human Placental Trophoblasts Confer Protection against Zika Virus Infection. Cell Host Microbe 2016, 19, 705-712, doi:10.1016/j.chom.2016.03.008.

59. Resources, B. Product Information Sheet for NR-50355. Collection, A.T.C., Ed. 2016.

60. Bayer, A.; Lennemann, Nicholas J.; Ouyang, Y.; Bramley, John C.; Morosky, S.; Marques, Ernesto Torres De A.; Cherry, S.; Sadovsky, Y.; Coyne, Carolyn B. Type III Interferons Produced by Human Placental Trophoblasts Confer Protection against Zika Virus Infection. Cell Host Microbe 2016, 19, 705-712, doi:https://doi.org/10.1016/j.chom.2016.03.008.

61. Loe, M.W.C.; Lee, R.C.H.; Chu, J.J.H. Antiviral activity of the FDA-approved drug candesartan cilexetil against Zika virus infection. Antiviral Research 2019, 172, 104637, doi:https://doi.org/10.1016/j.antiviral.2019.104637.

62. Lin, S.-C.; Chen, M.-C.; Liu, S.; Callahan, V.M.; Bracci, N.R.; Lehman, C.W.; Dahal, B.; de la Fuente, C.L.; Lin, C.-C.; Wang, T.T., et al. Phloretin inhibits Zika virus infection by interfering with cellular glucose utilisation. International Journal of Antimicrobial Agents 2019, 54, 80-84, doi:https://doi.org/10.1016/j.ijantimicag.2019.03.017. 
63. Tabata, T.; Petitt, M.; Puerta-Guardo, H.; Michlmayr, D.; Wang, C.; Fang-Hoover, J.; Harris, E.; Pereira, L. Zika Virus Targets Different Primary Human Placental Cells, Suggesting Two Routes for Vertical Transmission. Cell Host Microbe 2016, 20, 155-166, doi:https://doi.org/10.1016/j.chom.2016.07.002.

64. Technologies, S. STEMdiff Cerebral Organoid Kit. 1_2_0 ed.; Technologies, S., Ed. StemCell Technologies: Vancouver, 2020; Vol. DX21849.

65. Aldo, P.; You, Y.; Szigeti, K.; Horvath, T.L.; Lindenbach, B.; Mor, G. HSV-2 enhances ZIKV infection of the placenta and induces apoptosis in first-trimester trophoblast cells. American Journal of Reproductive Immunology 2016, 76, 348-357, doi:10.1111/aji.12578.

66. Zare Mehrjardi, M.; Shobeirian, F. The role of the placenta in prenatally acquired Zika virus infection. Virusdisease 2017, 28, 247-249, doi:10.1007/s13337-017-0399-z.

67. Vianna, R.A.d.O.; Rua, E.C.; Fernandes, A.R.; dos Santos, T.C.S.; Dalcastel, L.A.B.; dos Santos, M.L.B.; de Paula, P.d.S.; de Carvalho, F.R.; Pache de Faria, A.d.O.; Almeida, P.L., et al. Experience in diagnosing congenital Zika syndrome in Brazilian children born to asymptomatic mothers. Acta Tropica 2020, 206, 105438, doi:https://doi.org/10.1016/j.actatropica.2020.105438.

68. Cugola, F.R.; Fernandes, I.R.; Russo, F.B.; Freitas, B.C.; Dias, J.L.M.; Guimarães, K.P.; Benazzato, C.; Almeida, N.; Pignatari, G.C.; Romero, S., et al. The Brazilian Zika virus strain causes birth defects in experimental models. Nature 2016, 534, 267-271, doi:10.1038/nature18296.

69. Schwarz, E.R.; Oliveira, L.J.; Bonfante, F.; Pu, R.; Pozor, M.A.; Maclachlan, N.J.; Beachboard, S.; Barr, K.L.; Long, M.T. Experimental Infection of Mid-Gestation Pregnant Female and Intact Male Sheep with Zika Virus. Viruses 2020, 12, 291, doi:10.3390/v12030291.

70. Lenglet, Y.; Barau, G.; Robillard, P.Y.; Randrianaivo, H.; Michault, A.; Bouveret, A.; Gérardin, P.; Boumahni, B.; Touret, Y.; Kauffmann, E., et al. Chikungunya infection in pregnancy: Evidence for intrauterine infection in pregnant women and vertical transmission in the parturient. Survey of the Reunion Island outbreak. Journal de Gynecologie Obstetrique et Biologie de la Reproduction 2006, 35, 578583.

71. Touret, Y.; Randrianaivo, H.; Michault, A.; Schuffenecker, I.; Kauffmann, E.; Lenglet, Y.; Barau, G.; Fourmaintraux, A. Early maternal-fetal transmission of the Chikungunya virus. Presse Medicale 2006, 35, 1656-1658, doi:Pm-11-2006-35-11-c1-0755-4982-101019-200608574.

72. Watanaveeradej, V.; Endy, T.P.; Simasathien, S.; Kerdpanich, A.; Polprasert, N.; Aree, C.; Vaughn, D.W.; Nisalak, A. The study transplacental chikungunya virus antibody kinetics, Thailand. Emerg Infect Dis 2006, 12, 1770-1772, doi:10.3201/eid1211.051560 10.3201/eid1211.051560.

73. Chen, C.I.; Clark, D.C.; Pesavento, P.; Lerche, N.W.; Luciw, P.A.; Reisen, W.K.; Brault, A.C. Comparative pathogenesis of epidemic and enzootic Chikungunya viruses in a pregnant Rhesus macaque model. The American journal of tropical medicine and hygiene 2010, 83, 1249-1258, doi:10.4269/ajtmh.2010.10-0290 10.4269/ajtmh.2010.10-0290.

74. Jia, Q.; Jia, C.; Liu, Y.; Yang, Y.; Qi, J.; Tong, L.; Chen, H.; Zhang, M.; Che, J.; Li, B., et al. Clinical evidence for the immunogenicity and immune persistence of vaccination with yellow fever virus strain 17D in Chinese peacekeepers deployed to Africa. Antiviral Research 2019, 162, 1-4, doi:https://doi.org/10.1016/j.antiviral.2018.12.001.

75. Staples, J.E.; Monath, T.P.; Gershman, M.D.; Barrett, A.D.T. 63 - Yellow Fever Vaccines. In Plotkin's Vaccines (Seventh Edition), Plotkin, S.A., Orenstein, W.A., Offit, P.A., Edwards, K.M., Eds. Elsevier: 2018; https://doi.org/10.1016/B978-0-323-35761-6.00063-8pp. 1181-1265.e1120. 
76. Kiskova, T.; Mytsko, Y.; Schepelmann, M.; Helmer, H.; Fuchs, R.; Miedl, H.; Wadsack, C.; Ellinger, I. Expression of the neonatal Fc-receptor in placental-fetal endothelium and in cells of the placental immune system. Placenta 2019, 78, 36-43, doi:https://doi.org/10.1016/j.placenta.2019.02.012.

77. Simister, N.E.; Story, C.M. Human placental Fc receptors and the transmission of antibodies from mother to fetus. Journal of Reproductive Immunology 1997, 37, 1-23, doi:https://doi.org/10.1016/S01650378(97)00068-5.

78. Ellinger, I.; Schwab, M.; Stefanescu, A.; Hunziker, W.; Fuchs, R. IgG transport across trophoblastderived BeWo cells: a model system to study IgG transport in the placenta. European Journal of Immunology 1999, 29, 733-744, doi:10.1002/(sici)1521-4141(199903)29:03<733::Aid-immu733>3.0.Co;2-c.

79. Zimmerman, M.G.; Quicke, K.M.; O'Neal, J.T.; Arora, N.; Machiah, D.; Priyamvada, L.; Kauffman, R.C.; Register, E.; Adekunle, O.; Swieboda, D., et al. Cross-Reactive Dengue Virus Antibodies Augment Zika Virus Infection of Human Placental Macrophages. Cell Host Microbe 2018, 24, 731-742.e736, doi:https://doi.org/10.1016/j.chom.2018.10.008.

80. Brown, J.A.; Singh, G.; Acklin, J.A.; Lee, S.; Duehr, J.E.; Chokola, A.N.; Frere, J.J.; Hoffman, K.W.; Foster, G.A.; Krysztof, D., et al. Dengue Virus Immunity Increases Zika Virus-Induced Damage during Pregnancy. Immunity 2019, 50, 751-762.e755, doi:https://doi.org/10.1016/j.immuni.2019.01.005.

81. Wang, W.-H.; Urbina, A.N.; Wu, C.-C.; Lin, C.-Y.; Thitithanyanont, A.; Assavalapsakul, W.; Lu, P.-L.; Chen, Y.-H.; Wang, S.-F. An epidemiological survey of the current status of Zika and the immune interaction between dengue and Zika infection in Southern Taiwan. International Journal of Infectious Diseases 2020, 93, 151-159, doi:https://doi.org/10.1016/j.ijid.2020.01.031.

82. Pierson, T.C. Modeling antibody-enhanced dengue virus infection and disease in mice: protection or pathogenesis? Cell Host Microbe 2010, 7, 85-86, doi:10.1016/j.chom.2010.02.004 10.1016/j.chom.2010.02.004.

83. Petitt, M.; Tabata, T.; Puerta-Guardo, H.; Harris, E.; Pereira, L. Zika virus infection of first-trimester human placentas: utility of an explant model of replication to evaluate correlates of immune protection ex vivo. Current Opinion in Virology 2017, 27, 48-56, doi:https://doi.org/10.1016/j.coviro.2017.11.008.

84. Evans-Gilbert, T. Vertically transmitted chikungunya, Zika and dengue virus infections: The pathogenesis from mother to fetus and the implications of co-infections and vaccine development. International Journal of Pediatrics and Adolescent Medicine 2019, https://doi.org/10.1016/j.ijpam.2019.05.004, doi:https://doi.org/10.1016/j.ijpam.2019.05.004.

85. Reyes, L.; Golos, T.G. Hofbauer Cells: Their Role in Healthy and Complicated Pregnancy. Front Immunol 2018, 9, 2628-2628, doi:10.3389/fimmu.2018.02628.

86. Jones, C.J.P.; Choudhury, R.H.; Aplin, J.D. Functional changes in Hofbauer cell glycobiology during human pregnancy. Placenta 2015, 36, 1130-1137, doi:https://doi.org/10.1016/j.placenta.2015.07.131.

87. Seval, Y.; Korgun, E.T.; Demir, R. Hofbauer Cells in Early Human Placenta: Possible Implications in Vasculogenesis and Angiogenesis. Placenta 2007, 28, 841-845, doi:https://doi.org/10.1016/j.placenta.2007.01.010.

88. King, N.J.C.; Teixeira, M.M.; Mahalingam, S. Zika Virus: Mechanisms of Infection During Pregnancy. Trends in Microbiology 2017, 25, 701-702, doi:https://doi.org/10.1016/j.tim.2017.05.005.

89. Maeki, T.; Tajima, S.; Ikeda, M.; Kato, F.; Taniguchi, S.; Nakayama, E.; Takasaki, T.; Lim, C.-K.; Saijo, M. Analysis of cross-reactivity between flaviviruses with sera of patients with Japanese encephalitis showed the importance of neutralization tests for the diagnosis of Japanese encephalitis. Journal of Infection and Chemotherapy 2019, 25, 786-790, doi:https://doi.org/10.1016/j.jiac.2019.04.003. 
16 of 16

90. Maciejewski, S.; Pierson, T.C. Cross-Reactive Flavivirus Antibody: Friend and Foe? Cell Host Microbe 2018, 24, 622-624, doi:https://doi.org/10.1016/j.chom.2018.10.016. 